Discrete Comput Geom 30:263-276 (2003)

DOI: $10.1007 / \mathrm{s} 00454-003-0008-5$

Geometry

\title{
Slicing the Pie
}

\author{
Dan Ismailescu \\ Department of Mathematics, Hofstra University, \\ Hempstead, NY 11549-1000, USA \\ matdpi@hofstra.edu
}

\begin{abstract}
Given a circular unit disc and $n$ lines such that any two of them intersect inside the disc, we show that there exists a cell of area at least $\pi / 4 n$.
\end{abstract}

\section{Introduction}

The result stated in the abstract was motivated by the following conjecture:

Conjecture 1 [2]. Let $n$ great circles in general position (i.e., no three having a point in common) divide the sphere into $m=n(n-1)+2$ spherical polygons of areas $b_{1}, b_{2}, \ldots, b_{m}$. Then $\max b_{i} / \min b_{i} \rightarrow \infty$ as $n \rightarrow \infty$.

Many interesting questions similar with the one above may be raised; we formulate some of them as open problems in a later section. Despite the fact that line arrangements in the plane and great circle arrangements on the sphere received a great deal of attention in the literature, to the best of our knowledge very little is known about the so-called "small face-big face problems." Loosely speaking, given an arrangement of curves on a surface, we may ask for the existence of a certain cell, the measure of which is either much smaller or much larger than the average. The reader may consult [1] and [5] to get a flavor of these problems.

In this paper we present one such result. We hope that this will stimulate further research in this interesting and difficult area.

In Section 2 we introduce the notions needed in what follows. In particular, in Section 2.2 we follow the presentation from [3]. In Section 3 we define the associated polygon of an arrangement of lines and prove an interesting property for this polygon. In Section 4 we present the proof of our main result and in Section 5 we make a few remarks and present a list of open problems. 


\section{Basic Definitions}

\subsection{Arrangements of Lines}

An arrangement of lines $\mathcal{A}$ is a finite family of $n=n(\mathcal{A})$ lines $L_{1}, L_{2}, \ldots, L_{n}$ in the Euclidean plane $\mathbf{E}^{2}$. If no three lines are concurrent we say that the arrangement is simple. Moreover, the arrangement $\mathcal{A}$ is said to be in general position if it is simple and no two lines of $\mathcal{A}$ are parallel. In what follows we mostly deal with line arrangements of this latter type.

With an arrangement $\mathcal{A}$, we associate the two-dimensional cell complex into which the lines decompose $\mathbf{E}^{2}$. It is easy to prove that an arrangement in general position determines $\left(\begin{array}{l}n \\ 2\end{array}\right)$ vertices, $n^{2}$ edges, and $1+\left(\begin{array}{c}n+1 \\ 2\end{array}\right)$ faces (cells), $2 n$ of which are unbounded.

For our purposes, it is convenient to consider the cells as open sets. We define the interior (exterior) of an arrangement of lines as being the union of all bounded (resp. unbounded) cells determined by the arrangement.

\subsection{The Winding Number}

Let $P$ be an oriented polygon and let $A$ be any point of $\mathbf{E}^{2} \backslash P$. Let $R=[A, B)$ be a ray which contains no vertex of $P$. For each edge $E_{j}$ of $P$ we define an index $\psi\left(R, E_{j}\right)$ as follows:

$\psi\left(R, E_{j}\right)= \begin{cases}0, & \text { if } R \text { does not intersect } E_{j} ; \\ 1, & \text { if } E_{j} \text { crosses } R \text { in a counterclockwise direction as viewed from } A ; \\ -1, & \text { if } E_{j} \text { crosses } R \text { in a clockwise direction as viewed from } A .\end{cases}$

Then the winding number $w(R, P)$ of $R$ with respect to $P$ is defined as

$$
w(R, P)=\sum_{j} \psi\left(R, E_{j}\right)
$$

where summation is considered over all the edges of $P$ (see Fig. 1).

Thus, $w(R, P)$ is the signed difference between the number of edges that cross $R$ in a counterclockwise direction and the number that cross $R$ in a clockwise direction. It is easy to see that $w(R, P)$ has the same value of all rays $R$ with endpoint $A$ that do not contain a vertex of $P$.

Therefore the value of $w(R, P)$ can more appropriately be denoted by $w(A, P)$. As a function of $A, w(A, P)$ is continuous and takes only integer values; hence it is constant as $A$ ranges over any cell $C$ of $P$. Denote its value for $A \in C$ by $w(C, P)$. The value of $w(C, P)$ is often called the density of the cell $C$ with respect to $P$. It is clear that if $A$ lies in the exterior of $P$, then $w(A, P)=0$.

Given an oriented polygon $P=V_{1} V_{2} \cdots V_{n}$ where each $V_{i}$ has cartesian coordinates $\left(x_{i}, y_{i}\right)$ for $i=1 \cdots n$, we define its generalized area $G(P)$ as

$$
G(P)=\frac{1}{2} \cdot\left|\sum_{i=1}^{n}\left(x_{i} y_{i+1}-x_{i+1} y_{i}\right)\right|
$$




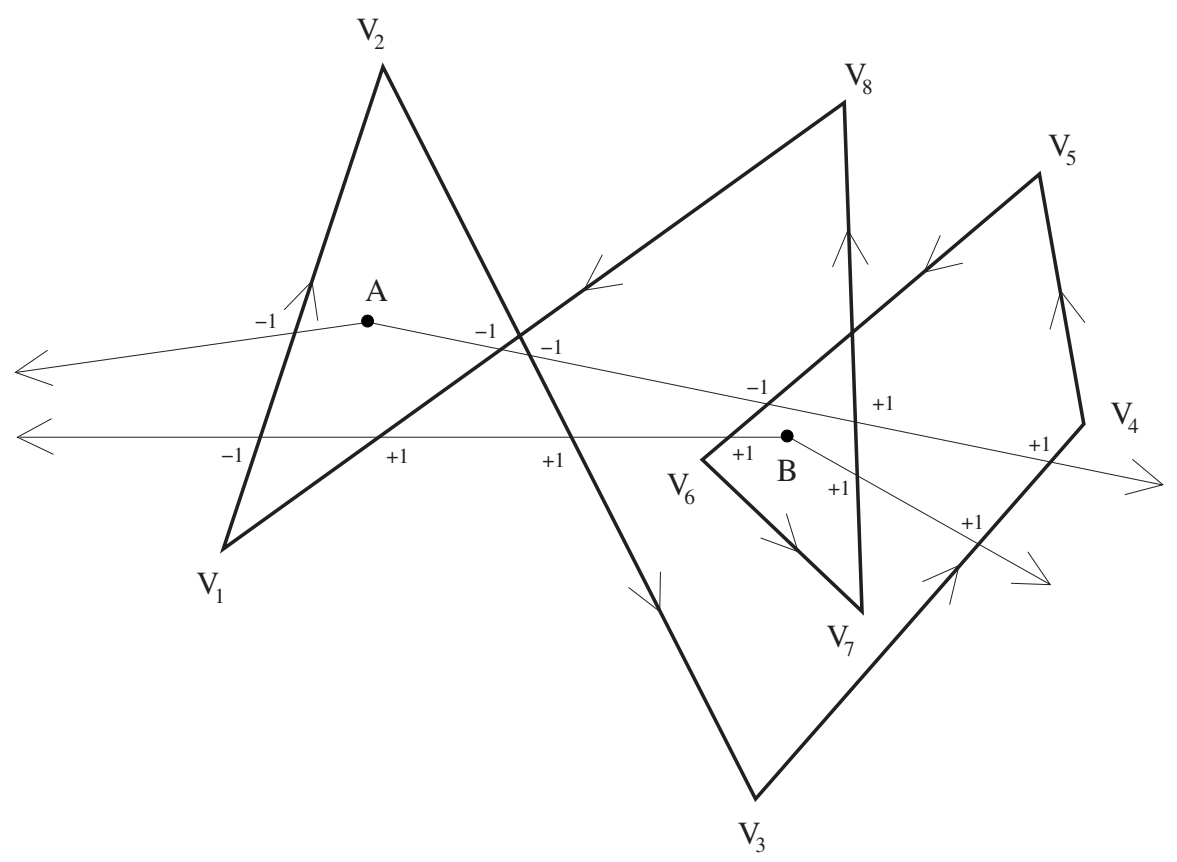

Fig. 1. For several rays issuing from $A$ and $B$, the values of $\psi$ are indicated near each intersection with the polygon $P$. In this example we have $w(A, P)=-1, w(B, P)=2$.

For simple polygons $G(P)$ is just the usual area. Here and in the following, unless otherwise stated, all subscripts will be taken modulo $n$. It is well known that for every oriented polygon $P$,

$$
G(P)=\left|\sum_{C} w(C, P) \cdot \operatorname{Area}(C)\right|,
$$

the summation being over all cells $C$ determined by $P$. This observation will be useful later.

\section{The Polygon Associated with an Arrangement of Lines}

Let $L_{1}, L_{2}, \ldots, L_{n}$ be the lines of an arrangement $\mathcal{A}$ sorted in decreasing order of their slopes, where $m_{i}$ is the slope of $L_{i}$. Hence,

$$
m_{1}>m_{2}>\cdots>m_{n-1}>m_{n} .
$$

Two lines will be called adjacent if they have consecutive subscripts. In particular, $L_{1}$ and $L_{n}$ are considered to be adjacent.

The vertices of the associated polygon $P(\mathcal{A})$ are the points determined by pairs of adjacent lines, $V_{i}=L_{i-1} \cap L_{i}$; the oriented edges are denoted by $E_{i}=\left[V_{i} V_{i+1}\right]$ (see Fig. 2) and the cartesian coordinates of $V_{i}$ are denoted by $x_{i}$ and $y_{i}$.

Our first result concerns a nice property of the associated polygon. 


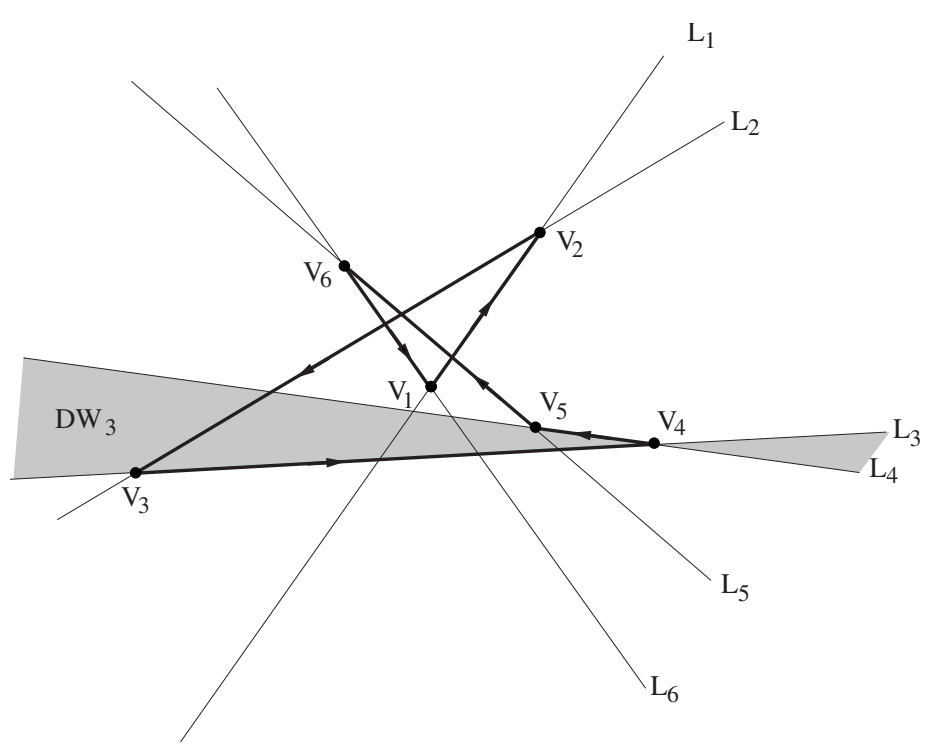

Fig. 2. The associated polygon $P(\mathcal{A})$ has a positive winding number with respect to each point contained in the interior of $\mathcal{A}$.

Theorem 1. The winding number of every interior point of an arrangement of lines $\mathcal{A}$ with respect to the associated polygon $P(\mathcal{A})$ is at least 1.

Proof. Let $L_{i}$ and $L_{i+1}$ be two adjacent lines of the arrangement and let $D W_{i}$ be the open double-wedge determined by these two lines (see Fig. 2). Using induction, it is easy to prove that for any arrangement with at least three lines we have

$$
\bigcup_{i=1}^{n} D W_{i} \supseteq \mathbf{E}^{2} \backslash \bigcup_{i=1}^{n} L_{i} .
$$

Let $O$ be an interior point of $\mathcal{A}$. We have to show that $w(O, P(\mathcal{A})) \geq 1$. From (4) it follows that $O$ is contained in some double-wedge $D W_{i}$. After an appropriate rigid motion we can assume that $O$ is at the origin, is contained in the upper angle of $D W_{n}$, and that $D W_{n}$ has a vertical symmetry axis. The possibilities are described in Fig. 3; in all four cases, the fact that $O$ belongs to the upper part of the double-wedge $D W_{n}$ implies that

$$
y_{1}-m_{1} x_{1}<0 \quad \text { and } \quad y_{n}-m_{n} x_{n}<0 .
$$

We compute $w(O, P(\mathcal{A}))$ by analyzing the intersections between the edges of $\mathcal{A}$ and the vertical halfline $O y$. One may also assume that no vertex of $P(\mathcal{A})$ lies on the vertical halfline $O y$ since otherwise one may slightly perturb point $O$ in such a way that it remains within the original cell while $O y$ contains no vertex, which means that none of the $x_{i}$ 's is zero. Moreover, since $O$ does not belong to any of the lines $L_{i}$, it follows that all quantities $y_{i}-m_{i} x_{i}$ are nonzero. 

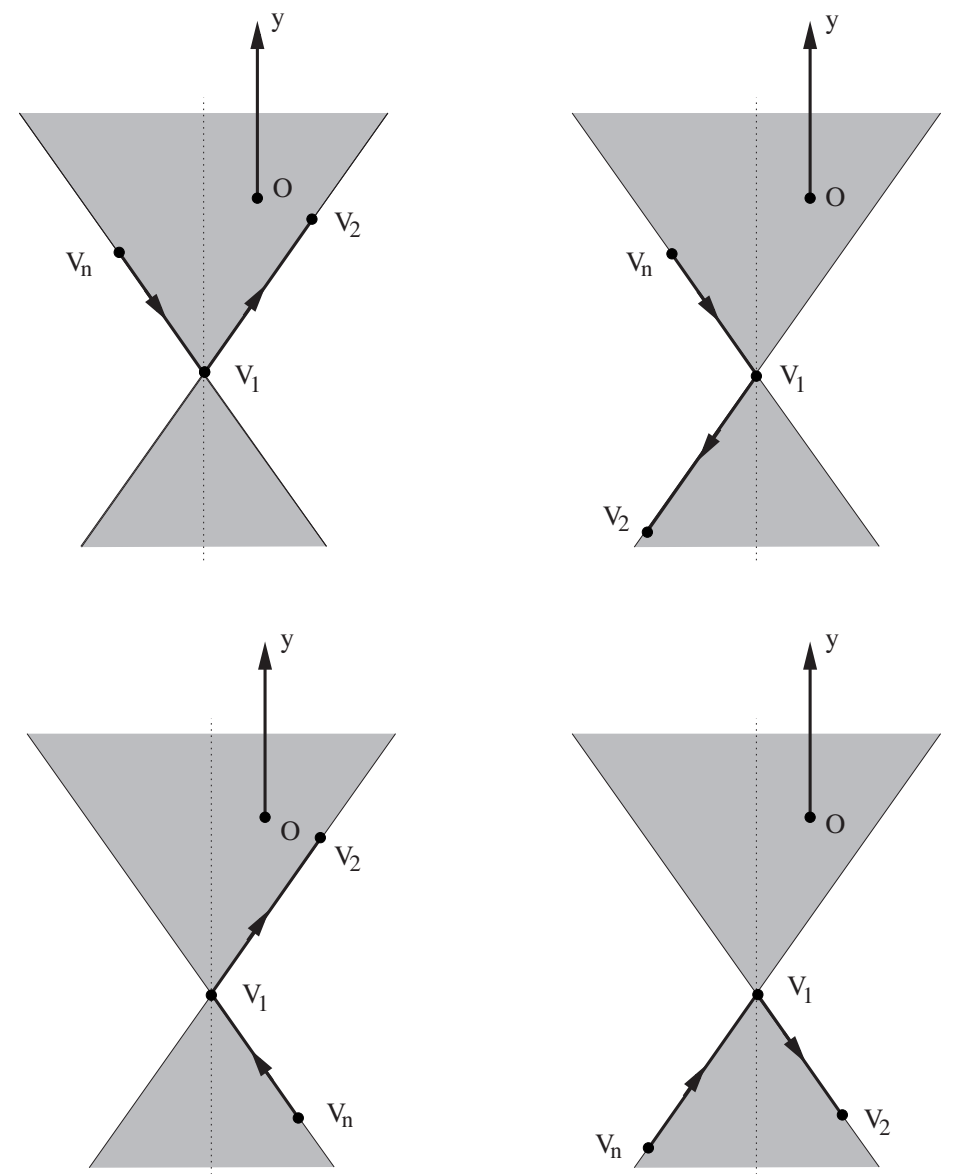

Fig. 3. The first and the last edges of the associated polygon.

For now, we first express the index $\psi\left(O y, E_{i}\right)$ in terms of the coordinates of the endpoints of $E_{i}, V_{i}$ and $V_{i+1}$ :

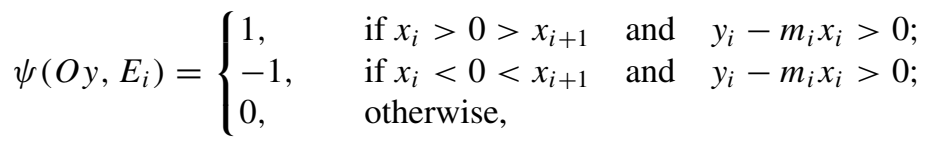

or in a more compact form

$$
\psi\left(O y, E_{i}\right)=\frac{1}{4}\left[\operatorname{sgn}\left(y_{i}-m_{i} x_{i}\right)+1\right] \cdot\left[\operatorname{sgn}\left(x_{i}\right)-\operatorname{sgn}\left(x_{i+1}\right)\right] .
$$

It then follows that

$4 w(O, P(\mathcal{A}))=\sum_{i=1}^{n} \operatorname{sgn}\left(y_{i}-m_{i} x_{i}\right) \cdot\left[\operatorname{sgn}\left(x_{i}\right)-\operatorname{sgn}\left(x_{i+1}\right)\right]+\sum_{i=1}^{n}\left[\operatorname{sgn}\left(x_{i}\right)-\operatorname{sgn}\left(x_{i+1}\right)\right]$. 
The second sum is obviously zero and after regrouping the terms in the first sum we obtain

$$
4 w(O, P(\mathcal{A}))=\sum_{i=1}^{n}\left\{\operatorname{sgn}\left[x_{i+1}\left(y_{i+1}-m_{i+1} x_{i+1}\right)\right]-\operatorname{sgn}\left[x_{i+1}\left(y_{i}-m_{i} x_{i}\right)\right]\right\} .
$$

Now, since $y_{i+1}=y_{i}+m_{i}\left(x_{i+1}-x_{i}\right)$ the above relation becomes

$4 w(O, P(\mathcal{A}))=\sum_{i=1}^{n}\left\{\operatorname{sgn}\left[x_{i+1}\left(y_{i}-m_{i} x_{i}\right)+x_{i+1}^{2}\left(m_{i}-m_{i+1}\right)\right]-\operatorname{sgn}\left[x_{i+1}\left(y_{i}-m_{i} x_{i}\right)\right]\right\}$

which, given the fact that $m_{i}>m_{i+1}$ for all $i \in\{1,2, \ldots, n-1\}$, implies that the first $n-1$ terms of the above sum are all nonnegative. The last term is $\operatorname{sgn}\left[x_{1}\left(y_{1}-m_{1} x_{1}\right)\right]-$ $\operatorname{sgn}\left[x_{1}\left(y_{n}-m_{n} x_{n}\right)\right]$ which is exactly zero by (5).

Therefore, the sum in (6) consists only of nonnegative terms and the only way $w(O, P(\mathcal{A}))$ can be zero is when all its terms are vanishing. This happens when, for every $i \in\{1,2, \ldots, n\},\left(y_{i+1}-m_{i+1} x_{i+1}\right)$ and $\left(y_{i}-m_{i} x_{i}\right)$ have the same sign. From this and (5) it follows that for all $i \in\{1,2, \ldots, n\}$ we have $y_{i}-m_{i} x_{i}<0$ which is equivalent to the fact that none of the lines $L_{i}$ intersects the halfline $O y$; consequently, in this case $O$ is an exterior point of the arrangement $\mathcal{A}$ contradicting our initial assumption. This ends the proof.

\section{The Main Result}

Theorem 2. Given a circular disc of unit radius and an arrangement $\mathcal{A}$ of $n$ lines such that all intersection points lie inside the disc, there exists a cell of area at least $\pi / 4 n$.

Proof. Obviously we can assume that no three lines are concurrent; otherwise we can slightly perturb the lines so that the areas of the cells are within $\varepsilon$ of their initial value (for $\varepsilon$ arbitrarily small) and the lines are in general position.

Here, the cells are the $1+n(n+1) / 2$ bounded connected components of $\mathbf{E}^{2}$ resulting when the lines and the circle are removed.

The main idea is to prove that the sum of the areas of the cells which have a circular arc as part of their boundary is "big," more precisely at least half of the area of the entire circular disc. Since there are $2 n$ such cells the claim in the theorem follows immediately. Equivalently, we will show that

$$
\sum_{C} \operatorname{Area}(C) \leq \frac{\pi}{2}
$$

where the sum is over all bounded polygonal cells $C$ determined by the arrangement $\mathcal{A}$.

Denote the lengths of the arcs determined by the lines $L_{1}, L_{2}, \ldots, L_{n}$ on the circle by $2 a_{1}, \ldots, 2 a_{2 n}$ as in Fig. 4 . Clearly, $0<a_{j}<\pi$ for all $j=1, \ldots, n$ and $\sum_{j=1}^{2 n} a_{j}=$ $\pi$. Using (1) it follows after some tedious but straightforward computations that the generalized area of the associated polygon $P(\mathcal{A})=V_{1} V_{2} \cdots V_{n}$ can be written as

$$
G(P(\mathcal{A}))=\left|\sum_{i=1}^{n} B_{i}\right|,
$$




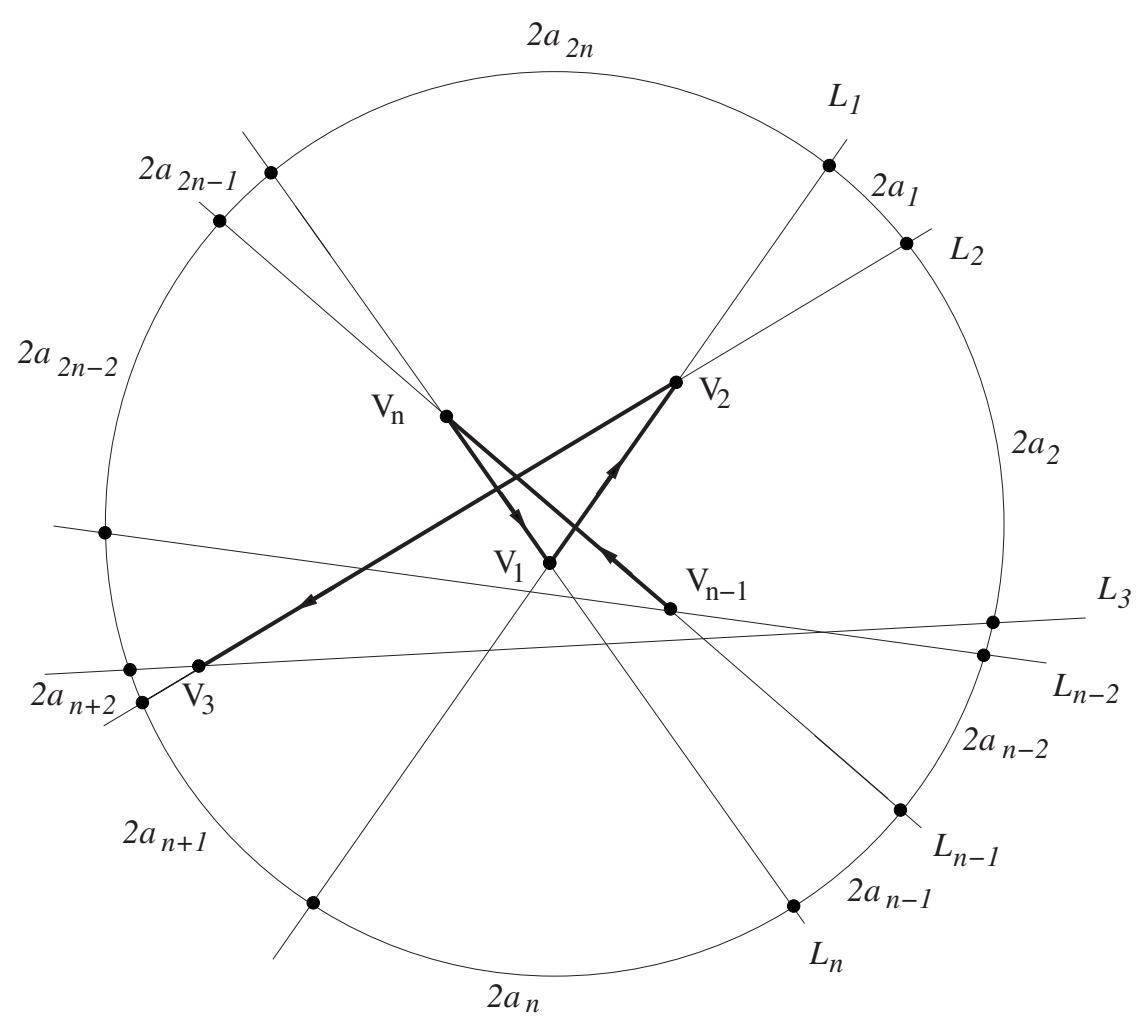

Fig. 4. The lines $L_{1}, L_{2}, \ldots, L_{n}$ pairwisely intersecting inside the unit circle.

where for each integer $1 \leq i \leq n$ we define

$B_{i}=$

$\frac{2 \sin a_{i} \sin a_{n+i}+\left(\sin ^{2} a_{i}+\sin ^{2} a_{n+i}\right) \cdot \cos \left(a_{i}+2 a_{i+1}+\cdots+2 a_{n+i-1}+a_{n+i}\right)}{2 \sin \left(a_{i}+a_{n+i}\right)}$.

We postpone the proof of equality (8) until Section 6.

We will need the following:

Claim. With $a_{j}, j=1, \ldots, 2 n$, and $B_{i}, i=1, \ldots, n$, defined as above we have

$$
-\sin \left(\frac{a_{i}+a_{n+i}}{2}\right) \leq B_{i} \leq \frac{\sin \left(a_{i}+a_{n+i}\right)}{2} .
$$

Proof. For proving the left inequality we notice first that

$$
B_{i} \geq \frac{2 \sin a_{i} \sin a_{n+i}-\left(\sin ^{2} a_{i}+\sin ^{2} a_{n+i}\right)}{2 \sin \left(a_{i}+a_{n+i}\right)}=-\frac{\left(\sin a_{i}-\sin a_{n+i}\right)^{2}}{2 \sin \left(a_{i}+a_{n+i}\right)} .
$$


It would be thus sufficient to show that

$$
\left(\sin a_{i}-\sin a_{n+i}\right)^{2} \leq 2 \sin \left(a_{i}+a_{n+i}\right) \sin \left(\frac{a_{i}+a_{n+i}}{2}\right)
$$

which is equivalent to

$$
\sin ^{2}\left(\frac{a_{i}-a_{n+i}}{2}\right) \cos \left(\frac{a_{i}+a_{n+i}}{2}\right) \leq \sin ^{2}\left(\frac{a_{i}+a_{n+i}}{2}\right) .
$$

However, since $a_{i}, a_{n+i} \in(0, \pi)$ we have the stronger inequality

$$
0 \leq \sin a_{i} \sin a_{n+i}=\sin ^{2}\left(\frac{a_{i}+a_{n+i}}{2}\right)-\sin ^{2}\left(\frac{a_{i}-a_{n+i}}{2}\right) .
$$

The right inequality will follow from the stronger

$$
B_{i} \leq \frac{\sin \left(a_{i}+a_{n+i}\right) \cos \left(a_{i}-a_{n+i}\right)}{2} .
$$

After eliminating the denominator this can be written in the equivalent form

$$
\begin{aligned}
\sin ^{2}\left(a_{i}\right. & \left.+a_{n+i}\right) \cos \left(a_{i}-a_{n+i}\right)-2 \sin a_{i} \sin a_{n+i} \\
& \geq\left(\sin ^{2} a_{i}+\sin ^{2} a_{n+i}\right) \cos \left(a_{i}+2 a_{i+1}+\cdots+2 a_{n+i-1}+a_{n+i}\right) .
\end{aligned}
$$

The left-hand side of the above inequality can be written as

$$
\cos \left(a_{i}-a_{n+i}\right)-\cos ^{2}\left(a_{i}+a_{n+i}\right) \cos \left(a_{i}-a_{n+i}\right)-\cos \left(a_{i}-a_{n+i}\right)+\cos \left(a_{i}+a_{n+i}\right)
$$

which after simplifying the similar terms becomes

$\cos \left(a_{i}+a_{n+i}\right) \cdot\left[1-\cos \left(a_{i}+a_{n+i}\right) \cos \left(a_{i}-a_{n+i}\right)\right]=\cos \left(a_{i}+a_{n+i}\right) \cdot\left(\sin ^{2} a_{i}+\sin ^{2} a_{n+i}\right)$.

Therefore, (10) is equivalent to proving that

$$
\left(\sin ^{2} a_{i}+\sin ^{2} a_{n+i}\right)\left(\cos \left(a_{i}+a_{n+i}\right)-\cos \left(a_{i}+2 a_{i+1}+\cdots+2 a_{n+i-1}+a_{n+i}\right)\right) \geq 0
$$

which reduces to showing that

$$
\begin{aligned}
& \left(\sin ^{2} a_{i}+\sin ^{2} a_{n+i}\right) \sin \left(a_{i+1}+\cdots+a_{n+i-1}\right) \\
& \quad \cdot \sin \left(a_{i}+a_{i+1}+\cdots+a_{n+i-1}+a_{n+i}\right) \geq 0 .
\end{aligned}
$$

The last inequality holds by our hypothesis assumptions. This ends the proof of the claim.

Returning now to the proof of the theorem, we first notice that the above claim immediately implies that

$$
-\frac{\pi}{2}<\sum_{i=1}^{n} B_{i}<\frac{\pi}{2}
$$


Indeed, by using Jensen's inequality for the sine function on $(0, \pi)$ and the claim we obtain

$$
\sum_{i=1}^{n} B_{i} \leq \frac{1}{2} \sum_{i=1}^{n} \sin \left(a_{i}+a_{n+i}\right) \leq \frac{n}{2} \cdot \sin \frac{\pi}{n}<\frac{\pi}{2} .
$$

Similarly,

$$
\sum_{i=1}^{n} B_{i} \geq-\sum_{i=1}^{n} \sin \left(\frac{a_{i}+a_{n+i}}{2}\right) \geq-n \cdot \sin \frac{\pi}{2 n}>-\frac{\pi}{2} .
$$

From (8) it follows that $G(P(\mathcal{A}))<\pi / 2$. Now using (2) and Theorem 1 we obtain

$$
\sum_{C} \operatorname{Area}(C) \leq \sum_{C} w(C, P) \cdot \operatorname{Area}(C)<\frac{\pi}{2},
$$

where the summation is over all bounded cells $C$ determined by $P(\mathcal{A})$. This proves (7) and with it the entire theorem.

\section{Conclusions and Open Problems}

The above result is optimal, up to a constant multiplicative factor; this can be easily seen by considering the long diagonals of a regular $2 n$-gon as the $n$ lines of our arrangement. In this case all cells have area $\leq \pi / 2 n$.

As an immediate consequence, if the lines in Theorem 2 are in general position and $b_{1}, b_{2}, \ldots, b_{p}$ denote the areas of the $p=1+n(n-1) / 2$ cells, we have

$$
\frac{\max b_{i}}{\min b_{i}} \geq \frac{\pi / 4 n}{\pi /[1+n(n+1) / 2]} \geq \frac{n+1}{8} .
$$

An attempt to generalize our result is given by the following:

Conjecture 2. An arrangement of $n$ lines in general position in the plane determines $s=\left(\begin{array}{c}n-1 \\ 2\end{array}\right)$ bounded cells of areas $b_{1}, b_{2}, \ldots, b_{s}$. Then $\max b_{i} / \min b_{i} \rightarrow \infty$ as $n \rightarrow \infty$.

The major difference between this conjecture and Theorem 2 is the absence of good candidates for the role of "big cells." New ideas seem to be needed. We can eventually try to generalize the above conjecture for the case of pseudolines, but this, if true, seems to be hopelessly difficult. Instead, maybe the following problem has a much better chance to be solved first.

Conjecture 3. Let $f_{1}, f_{2}, \ldots, f_{n-1}:[0, \pi] \rightarrow(-1,1)$ ben-1 differentiable functions satisfying the following properties:

(a) For every $1 \leq i \leq n-1$, the function $f_{i}$ has at most one local extrema in $(0, \pi)$.

(b) For every pair of indices $1 \leq i<j \leq n-1$, the equation $f_{i}(x)=f_{j}(x)$ has exactly one solution; the graphs properly cross at this point.

(c) For every three distinct indices $1 \leq i<j<k \leq n-1$, the equation $f_{i}(x)=$ $f_{j}(x)=f_{k}(x)$ has no solution. 
Then, if $b_{1}, b_{2}, \ldots, b_{p}$ denote the areas of the $p=1+n(n-1) / 2$ cells in which the rectangle $[0, \pi] \times[-1,1]$ is divided by the graphs of the functions $f_{1}, f_{2}, \ldots, f_{n-1}$, we have $\max b_{i} / \min b_{i} \rightarrow \infty$ as $n \rightarrow \infty$.

It is interesting to note that Conjecture 3 is stronger than the original conjecture of L. Fejes Tóth mentioned in the Introduction. The argument is sketched below.

Given $n$ great circles in general position on a unit sphere $S$ start by choosing a diameter that belongs to exactly one of these circles; with no loss of generality assume that this diameter is vertical. Then let $C$ be the vertical cylinder of height 2 tangent to $S$. Then $S$ and $C$ touch in the horizontal great circle $E$ on the sphere $S$, which is the equator of $S$; we can assume that $E$ is not one of the initial great circles. Let $a$ and $b$ be the corresponding north and south poles of $S$; the straight vertical segment $a b$ is the common axis of $S$ and $C$. Let $x$ be any point of $S$, other than the poles $a$ and $b$. There is then a unique point $y$ on the axis $a b$ at the same height as $x$. When the horizontal segment $y x$ is produced beyond $x$, its continuation will cut the cylinder $C$ at some point $z$, at the same height as $x$ and $y$. We call $z$ the axial projection of $x$, see Fig. 5(a)). This is the famous Archimedean projection and it is a well-known fact that it preserves area (a nice elementary proof can be found in [4]).

By our choice, the image of the circle whose diameter was originally selected will consist of two vertical generators of the cylinder $C$ while all the other circles will project into centrally symmetric curves.

If $C$ is now slit open along one of the vertical generators mentioned above, it can be unrolled onto the plane as a rectangle of length $2 \pi$ and height 2 . Straightforward computations show that the images of the remaining $n-1$ great circles after unrolling can be expressed as graphs of functions of the following form, $f_{\alpha, \beta}:[0,2 \pi] \rightarrow(-1,1)$, where $\alpha \in[0,2 \pi), \beta>0$, and

$$
f_{\alpha, \beta}(x)=\frac{\cos (x-\alpha)}{\sqrt{\cos ^{2}(x-\alpha)+\beta^{2}}} .
$$

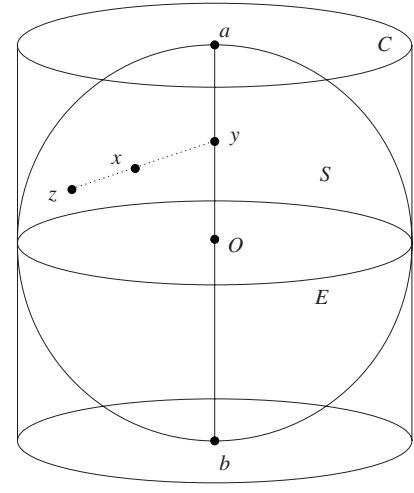

(a)

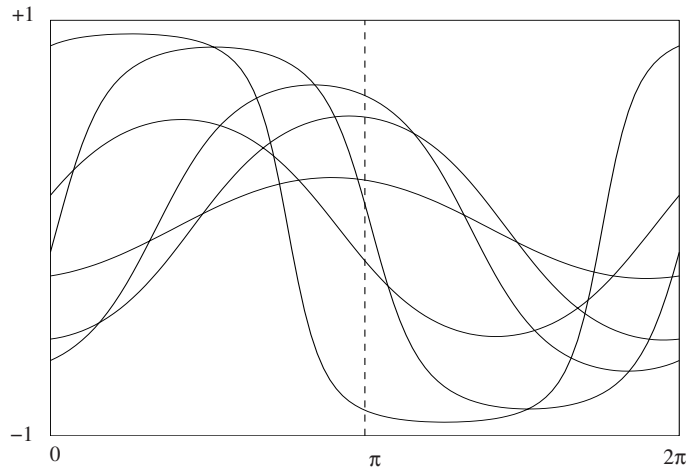

(b)

Fig. 5. (a) The Archimedean projection. (b) A family of seven great circles after projection and unrolling; the vertical circle projects onto the boundary and the dotted symmetry axis of the rectangle. 
An example of such a family of functions is presented in Fig. 5(b)). Obviously, given the symmetry it is enough to consider just the interval $[0, \pi]$. It is easy to check that the functions restricted to this interval have the properties mentioned in the hypothesis of Conjecture 3.

\section{Proof of (8)}

For every $1 \leq i \leq n$, denote by $A_{i}$ and $A_{n+i}$ the points in which line $L_{i}$ intersects the circle (see Fig. 6). Also, for every $1 \leq i \leq n$, we introduce the following notations:

$$
\begin{aligned}
b_{i} & =a_{i+1}+a_{i+2}+\cdots+a_{n+i-3}+a_{n+i-2}, \\
b_{n+i} & =a_{n+i+1}+\cdots+a_{2 n}+a_{1}+\cdots+a_{i-2} .
\end{aligned}
$$

In what follows all the subscripts of the quantities $a_{k}$ and $b_{k}$ are taken modulo $2 n$. Recall that $x_{i}$ and $y_{i}$ represent the cartesian coordinates of the vertex $V_{i}$. We want to express $x_{i} y_{i+1}-x_{i+1} y_{i}$ in terms of $a_{i-1}, a_{i}, b_{i}, a_{n+i-1}, a_{n+i}$, and $b_{n+i}$.

Denote by $\lambda_{i}$, respectively $\mu_{i}$, the ratios in which the point $V_{i}$, respectively $V_{i+1}$, divides the chord $A_{i} A_{n+i}$. That is,

$$
\lambda_{i}=\frac{\left|A_{i} V_{i}\right|}{\left|A_{i} A_{n+i}\right|} \quad \text { and } \quad \mu_{i}=\frac{\left|A_{i} V_{i+1}\right|}{\left|A_{i} A_{n+i}\right|} .
$$

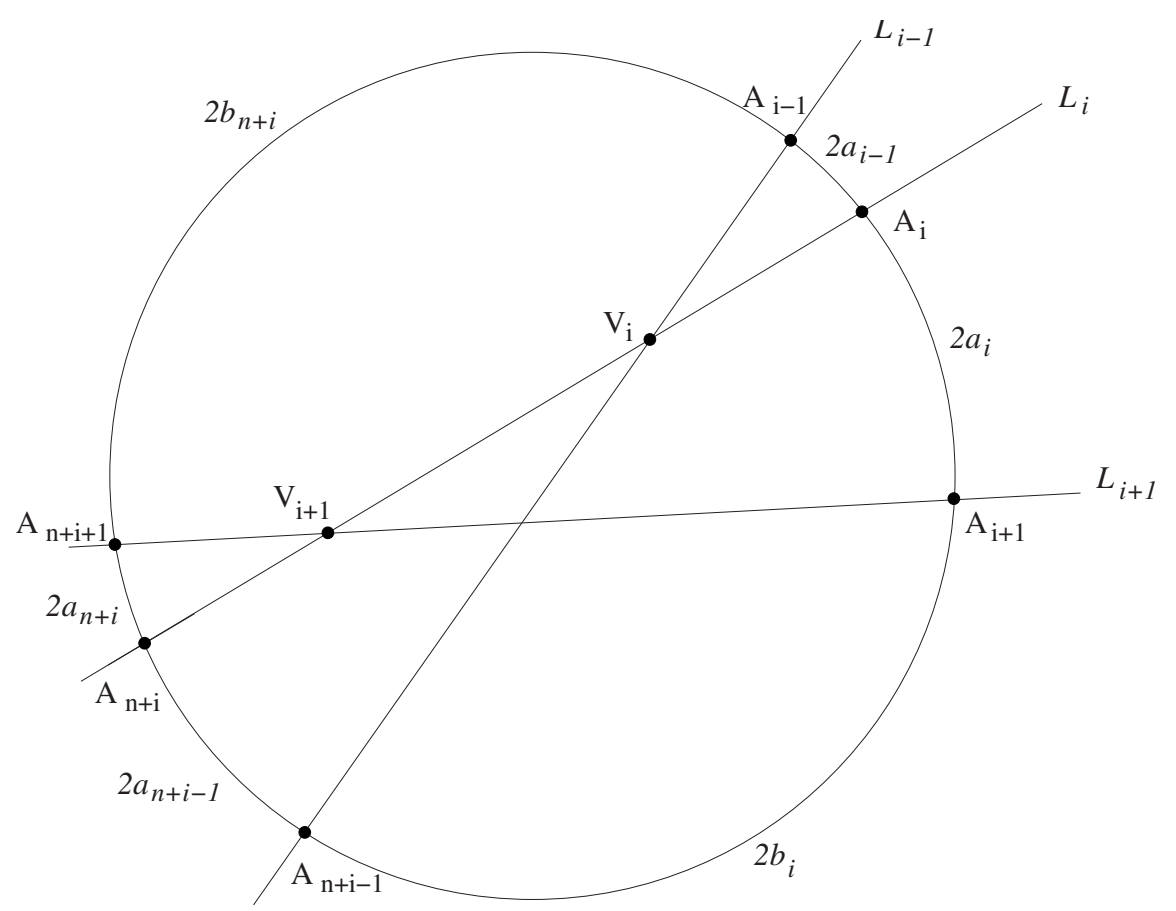

Fig. 6. Three consecutive lines $L_{i-1}, L_{i}$, and $L_{i+1}$ and the arcs determined by them on the circle. 
It follows that

$$
x_{i}=\lambda_{i} x_{A_{i}}+\left(1-\lambda_{i}\right) x_{A_{n+i}}, \quad y_{i}=\lambda_{i} y_{A_{i}}+\left(1-\lambda_{i}\right) y_{A_{n+i}}
$$

and, similarly,

$$
x_{i+1}=\mu_{i} x_{A_{i}}+\left(1-\mu_{i}\right) x_{A_{n+i}}, \quad y_{i+1}=\mu_{i} y_{A_{i}}+\left(1-\mu_{i}\right) y_{A_{n+i}} .
$$

A short computation gives that

$$
\begin{aligned}
x_{i} y_{i+1}-x_{i+1} y_{i} & =\left(\lambda_{i}-\mu_{i}\right)\left(x_{A_{i}} y_{A_{n+i}}-x_{A_{n+i}} y_{A_{i}}\right) \\
& =-\left(\lambda_{i}-\mu_{i}\right) \sin \left(2 a_{i}+2 b_{i}+2 a_{n+i-1}\right) .
\end{aligned}
$$

Applying the sine theorem to the triangles $A_{i} V_{i} A_{i-1}$ and $A_{i} A_{n+i} A_{i-1}$ we obtain

$$
\frac{\left|A_{i} V_{i}\right|}{\left|A_{i-1} A_{i}\right|}=\frac{\sin \left(a_{i}+b_{i}\right)}{\sin \left(a_{i-1}+a_{n+i-1}\right)}, \quad \text { respectively } \frac{\left|A_{i} A_{n+i}\right|}{\left|A_{i-1} A_{i}\right|}=\frac{\sin \left(a_{i}+b_{i}+a_{n+i-1}\right)}{\sin a_{i-1}}
$$

which implies that

$$
\lambda_{i}=\frac{\sin \left(a_{i}+b_{i}\right)}{\sin \left(a_{i-1}+a_{n+i-1}\right)} \cdot \frac{\sin a_{i-1}}{\sin \left(a_{i}+b_{i}+a_{n+i-1}\right)} .
$$

Similarly, the sine theorem applied to the triangles $A_{i} V_{i+1} A_{i+1}$ and $A_{i} A_{n+i} A_{i+1}$ gives $\frac{\left|A_{i} V_{i+1}\right|}{\left|A_{i+1} A_{i}\right|}=\frac{\sin \left(a_{i-1}+b_{n+i}\right)}{\sin \left(a_{i}+a_{n+i}\right)}, \quad$ respectively $\quad \frac{\left|A_{i} A_{n+i}\right|}{\left|A_{i+1} A_{i}\right|}=\frac{\sin \left(a_{i}+b_{i}+a_{n+i-1}\right)}{\sin a_{i}}$ from which

$$
\mu_{i}=\frac{\sin \left(a_{i-1}+b_{n+i}\right)}{\sin \left(a_{i}+a_{n+i}\right)} \cdot \frac{\sin a_{i}}{\sin \left(a_{i}+b_{i}+a_{n+i-1}\right)} .
$$

Using (15) and (16), equality (14) implies that

$$
\begin{aligned}
\frac{1}{2}\left(x_{i} y_{i+1}-x_{i+1} y_{i}\right)= & -\left(\lambda_{i}-\mu_{i}\right) \sin \left(a_{i}+b_{i}+a_{n+i-1}\right) \cos \left(a_{i}+b_{i}+a_{n+i-1}\right) \\
= & {\left[\frac{\sin a_{i} \sin \left(a_{i-1}+b_{n+i}\right)}{\sin \left(a_{i}+a_{n+i}\right)}-\frac{\sin a_{i-1} \sin \left(a_{i}+b_{i}\right)}{\sin \left(a_{i-1}+a_{n+i-1}\right)}\right] } \\
& \cdot \cos \left(a_{i}+b_{i}+a_{n+i-1}\right)
\end{aligned}
$$

Analogously, using the sine theorem in the triangles $A_{n+i} V_{i} A_{n+i-1}$ and $A_{n+i} A_{i}$ $A_{n+i-1}$, respectively $A_{n+i} V_{i+1} A_{n+i+1}$ and $A_{n+i} A_{i} A_{n+i+1}$, we obtain

$$
1-\lambda_{i}=\frac{\sin \left(a_{n+i}+b_{n+i}\right)}{\sin \left(a_{i-1}+a_{n+i-1}\right)} \cdot \frac{\sin a_{n+i-1}}{\sin \left(a_{i}+b_{i}+a_{n+i-1}\right)},
$$

respectively,

$$
1-\mu_{i}=\frac{\sin \left(a_{n+i-1}+b_{i}\right)}{\sin \left(a_{i}+a_{n+i}\right)} \cdot \frac{\sin a_{n+i}}{\sin \left(a_{i}+b_{i}+a_{n+i-1}\right)}
$$


Using (18) and (19), equality (14) becomes

$$
\begin{aligned}
\frac{1}{2}\left(x_{i} y_{i+1}-x_{i+1} y_{i}\right)= & {\left[\left(1-\lambda_{i}\right)-\left(1-\mu_{i}\right)\right] \sin \left(a_{i}+b_{i}+a_{n+i-1}\right) } \\
& \cdot \cos \left(a_{i}+b_{i}+a_{n+i-1}\right) \\
= & {\left[\frac{\sin a_{n+i-1} \sin \left(a_{n+i}+b_{n+i}\right)}{\sin \left(a_{i-1}+a_{n+i-1}\right)}-\frac{\sin a_{n+i} \sin \left(a_{n+i-1}+b_{i}\right)}{\sin \left(a_{i}+a_{n+i}\right)}\right] } \\
& \cdot \cos \left(a_{i}+b_{i}+a_{n+i-1}\right) .
\end{aligned}
$$

Adding (17) and (20) we obtain

$$
\begin{aligned}
x_{i} & y_{i+1}-x_{i+1} y_{i} \\
= & \frac{\left[\sin a_{i} \sin \left(a_{i-1}+b_{n+i}\right)-\sin a_{n+i} \sin \left(a_{n+i-1}+b_{i}\right)\right] \cos \left(a_{i}+b_{i}+a_{n+i-1}\right)}{\sin \left(a_{i}+a_{n+i}\right)} \\
& +\frac{\left[\sin a_{n+i-1} \sin \left(a_{n+i}+b_{n+i}\right)-\sin a_{i-1} \sin \left(a_{i}+b_{i}\right)\right] \cos \left(a_{i}+b_{i}+a_{n+i-1}\right)}{\sin \left(a_{i-1}+a_{n+i-1}\right)}
\end{aligned}
$$

After regrouping the terms with the same denominator, it follows that

$$
\frac{1}{2} \sum_{i=1}^{n}\left(x_{i} y_{i+1}-x_{i+1} y_{i}\right)=\sum_{i=1}^{n} \frac{p_{i}+q_{i}}{2 \sin \left(a_{i}+a_{n+i}\right)},
$$

where, for every $1 \leq i \leq n$,

$$
p_{i}=\left[\sin a_{i} \sin \left(a_{i-1}+b_{n+i}\right)-\sin a_{n+i} \sin \left(a_{n+i-1}+b_{i}\right)\right] \cos \left(a_{i}+b_{i}+a_{n+i-1}\right)
$$

and

$$
\begin{aligned}
q_{i}= & {\left[\sin a_{n+i} \sin \left(a_{n+i+1}+b_{n+i+1}\right)-\sin a_{i} \sin \left(a_{i+1}+b_{i+1}\right)\right] } \\
& \cdot \cos \left(a_{i+1}+b_{i+1}+a_{n+i}\right) .
\end{aligned}
$$

Therefore, for proving (8) it would suffice to show that

$$
\begin{aligned}
p_{i}+q_{i}= & 2 \sin a_{i} \sin a_{n+i}+\left(\sin ^{2} a_{i}+\sin ^{2} a_{n+i}\right) \\
& \cdot \cos \left(a_{i}+2 a_{i+1}+\cdots+2 a_{n+i-1}+a_{n+i}\right) .
\end{aligned}
$$

On one hand, expanding $p_{i}$ from (22) we obtain

$$
\begin{aligned}
& p_{i}=-\frac{1}{2} \sin a_{n+i} {\left[\sin \left(2 a_{n+i-1}+2 b_{i}+a_{i}\right)-\sin a_{i}\right] } \\
&+\frac{1}{2} \sin a_{i}[ \sin \left(a_{i-1}+a_{i}+b_{i}+a_{n+i-1}+b_{n+i}\right) \\
&\left.+\sin \left(a_{i-1}+b_{n+i}-a_{i}-b_{i}-a_{n+i-1}\right)\right]
\end{aligned}
$$

which, given that $b_{n+i}=\pi-\left(a_{i-1}+a_{i}+b_{i}+a_{n+i-1}+a_{n+i}\right)$, becomes

$$
\begin{aligned}
p_{i}= & \sin a_{i} \sin a_{n+i}+\frac{1}{2} \sin a_{i} \sin \left(2 a_{i}+2 b_{i}+2 a_{n+i-1}+a_{n+i}\right) \\
& -\frac{1}{2} \sin a_{n+i} \sin \left(a_{i}+2 b_{i}+2 a_{n+i-1}\right) .
\end{aligned}
$$


Similarly, expanding $q_{i}$ from (23) and using that $b_{n+i+1}=\pi-\left(a_{i}+a_{i+1}+b_{i+1}+\right.$ $\left.a_{n+i}+a_{n+i+1}\right)$ we obtain

$$
\begin{aligned}
q_{i}= & \sin a_{i} \sin a_{n+i}+\frac{1}{2} \sin a_{n+i} \sin \left(a_{i}+2 a_{i+1}+2 b_{i+1}+2 a_{n+i}\right) \\
& -\frac{1}{2} \sin a_{i} \sin \left(2 a_{i+1}+2 b_{i+1}+a_{n+i}\right) .
\end{aligned}
$$

Now combining (25) and (26) results in

$$
\begin{aligned}
p_{i}+q_{i}= & 2 \sin a_{i} \sin a_{n+i} \\
& +\frac{1}{2} \sin a_{i}\left[\sin \left(2 a_{i}+2 b_{i}+2 a_{n+i-1}+a_{n+i}\right)-\sin \left(2 a_{i+1}+2 b_{i+1}+a_{n+i}\right)\right] \\
& +\frac{1}{2} \sin a_{n+i}\left[\sin \left(a_{i}+2 a_{i+1}+2 b_{i+1}+2 a_{n+i}\right)\right. \\
& \left.\quad-\sin \left(a_{i}+2 b_{i}+2 a_{n+i-1}\right)\right]
\end{aligned}
$$

which after transforming the sums into products inside the square brackets becomes

$$
\begin{aligned}
p_{i}+q_{i}= & 2 \sin a_{i} \sin a_{n+i} \\
& +\sin a_{i} \sin \left(a_{i}+b_{i}+a_{n+i-1}-a_{i+1}-b_{i+1}\right) \\
& \cdot \cos \left(a_{i}+a_{i+1}+b_{i}+b_{i+1}+a_{n+i-1}+a_{n+i}\right) \\
& +\sin a_{n+i} \sin \left(a_{i+1}+b_{i+1}+a_{n+i}-b_{i}-a_{n+i-1}\right) \\
& \cdot \cos \left(a_{i}+a_{i+1}+b_{i}+b_{i+1}+a_{n+i-1}+a_{n+i}\right) .
\end{aligned}
$$

Now using (12) the above equality becomes

$$
\begin{aligned}
p_{i}+q_{i}= & 2 \sin a_{i} \sin a_{n+i}+\left(\sin ^{2} a_{i}+\sin ^{2} a_{n+i}\right) \\
& \cdot \cos \left(a_{i}+2 a_{i+1}+\cdots+2 a_{n+i-1}+a_{n+i}\right)
\end{aligned}
$$

which is exactly (24), the equality to be shown.

\section{References}

1. G. Barequet, A Duality between Small-Face Problems in Arrangements of Lines and Heilbronn-Type Problems, Discrete Mathematics, 237(1-3) (2001), 1-12.

2. L. Fejes Tóth, On Spherical Tilings Generated by Great Circles, Geometriae Dedicata, 23 (1987), 67-71.

3. B. Grünbaum and G. C. Shepard, Rotation and Winding Numbers for Planar Polygons and Curves, Transactions of the American Mathematical Society, 332(1) (1990), 169-187.

4. Z. A. Melzak, Invitation to Geometry, Pure and Applied Mathematics. Wiley-Interscience, New York, 1983, pp. $123-130$.

5. J. S. B. Mitchell, On the Existence of Small Faces in Arrangements of Lines, manuscript, 1995. (http:/www.ams.sunysb.edu/ jsbm/publications.html)

Received June 13, 2000, and in revised form February 21, 2001, and April 2, 2002.

Online publication July 15, 2003. 